\title{
Generating Knowledge on Networks in Environmental Governance
}

\author{
Marion Glaser \\ Social-Ecological Systems Analysis Working Group, Social Science Department, \\ Leibniz Centre for Tropical Marine Research, Bremen, Germany \\ Barbara Schröter ${ }^{1}$ \\ Governance of Ecosystem Services Working Group, Leibniz Centre for Agricultural \\ Landscape Research (ZALF), Müncheberg, Germany; Lund University, Centre for \\ Sustainability Studies (LUCSUS), Sweden
}

\section{Abstract}

This paper introduces the special issue on "Generating Sustainability-Supporting Knowledge on Social Networks in the Governance and Management of SocialEcological Systems." Understanding the interactions of actors and institutions is important for successful governance of human-nature relations. Social network analysis is able to capture and analyze these governance and management interactions, and there is a range of existing tools for collecting, visualizing, and modeling data on social and social-ecological networks. This introductory paper aims to give an overview of the collected papers as well as an outlook of new arising topics in the field. After presenting the single contributions to this special issue, we share our thoughts on network types and performance, network states and dynamics, limits of network studies, new conceptual focuses in network analysis, and methodological innovations (mixed methods and new tools). We conclude with a reflection of the contribution of this special issue to environmental governance.

Keywords: environmental management, social-ecological network analysis, socialecological systems, social network analysis

\section{Introduction}

For several decades now, sustainability research and environmental governance policies, along with diverse tools for environmental research and management, have advocated for-and undertaken - the integration of diverse actors from multiple system levels in systems analysis (Grimble \& Wellard, 1997; Phillipson et al., 2012; Reed, 2008; and a recent example, the global Future Earth project ${ }^{2}$ ).

\footnotetext{
Corresponding author: barbara.schroeter@zalf.de.

futureearth.org/2020/12/08/transition-update-1-scaling-up-global-sustainability-science/.
} 
Implicit in these efforts is a growing focus on social and social-ecological networks. Understanding the interactions of actors and/or organizations with each other and with their natural environment is an increasingly crucial ingredient for the effective governance of human-nature relations. This is a major challenge for academiain particular, for the fast-growing field of transdisciplinary sustainability science. There has been progress in methods such as agent-based modeling and network analysis but the scarcity of methods for the analysis of social-ecological systems, which was recognized over a decade ago (Glaeser et al., 2009), continues today. In view of increasing sustainability challenges, in particular the analysis of socialecological systems and their governance needs, it is essential to further engage with methodological innovations.

New methods for collecting, visualizing, and modeling data on social and socialecological networks in environmental governance and management are emerging, in particular quantitative, qualitative, and mixed methods that foster stakeholder participation and synergistic knowledge generation. In order to depict a spectrum of these innovations, this special issue focuses on the rapidly developing field of methods for network analysis (Kluger et al., 2020; Schwenke \& Holzkämper, this issue), which offers a range of innovative options across disciplines for the analysis of environmental governance.

The contributions to this special issue explore different methodological approaches for analyzing social and social-ecological networks relevant to the governance and management of social-ecological systems and present related exemplary results for environmental governance.

María Mancilla García and Örjan Bodin set out to differentiate to what extent power resides within network structures and whether it is rooted in actor attributes such as class and wealth. Through its methodologically innovative combination of social network analysis (SNA) with structural equation modeling and the qualitative analysis of open-ended interview data, this study informs the classic sociological debate, and Giddens's structuration theory in particular, on the roles of structure and agency. The authors focus on differences between the influence and the centrality of actors in an information exchange network for the governance of the East Brazilian Paraíba do Sul River, a network that spans three Brazilian states. The study explores correspondences and causal links between different statistical measures of centrality and measures of influence and popularity by using SNA.

Central findings are that high influence does not necessarily coincide with high social network centrality of a governance actor, and that influential actors with very different attributes can emerge. Neither formal position nor financial resources are found to entirely determine actors' influence, but the authors detect some scope for agency to change networks and influence levels. They conclude that since multiple forums with overlapping environmental governance competencies exist in their 
case study region, not only the relations within a network need to be analyzed, but also those between networks. The article offers a path toward a fine-grained, differentiated methodological approach for identifying the causal relations between network structures and positions and actors' influence. By testing a set of hypotheses with empirical data from a Brazilian regional case study on water governance, the article demonstrates the strategic use of networks while being well anchored in theoretical debates. This type of analysis has important potential for policy and practice in the emerging field of environmental governance.

Marco Scotti, Daniel Pereira, and Antonio Bodini offer an entirely method-focused article that presents loop analysis as a qualitative tool for linking disciplinary domains in integrated analyses of the natural and social science variables that are central for environmental governance and ecosystem-based management. Citing diverse case examples, they argue that loop analysis is particularly powerful for analyzing social-ecological systems for which data availability is poor. Adopting an interdisciplinary network perspective that includes ecology, economy, and society, the authors demonstrate that with its simple signed directed graphs, loop analysis is able to show the paths along which perturbations travel through a social-ecological system and identify the associated feedback structures and causal mechanisms in these complex systems. This helps, in their own words, to "make the arcane obvious" while the simple graph format of loop analysis also facilitates the participation of non-academic stakeholders in social-ecological systems model building. Given these important strengths, major remaining limitations of loop analysis concern nonlinearity, problems with the selection of temporal and spatial system levels, as well as with the timing of diverse system changes.

Marina Ribeiro Corrêa, Luciana Xavier, Eike Holzkämper, Mariana de Andrade, Alexander Turra, and Marion Glaser offer an applied study of social networks, examining the role and potential of public sector beach managers for advancing the ecosystem service-oriented management of the social-ecological systems associated with sandy beaches. The authors apply the Net-Map tool to the construction of both current and desired future beach governance networks from the perceptions of local beach managers in four municipalities on the northern coast of São Paulo State, Brazil. They then apply quantitative SNA methods to analyze their Net-Map data and obtain a set of quantitative metrics on beach managers' governance network perceptions. They find that local beach managers envision governance network transformation toward ecosystem-based beach management, and that they may act as effective and motivated leaders in this if supported in the development of skills and of a wider regional identity. The focus on the perceptions of local environmental managers that this study adopts is identified as a new way of fostering collaborative environmental governance to support the successful design and implementation of environmental governance. 
Philipp Gorris and Marion Glaser focus on the information transmission capacity and the robustness of actor networks in different approaches to collaborative governance of coastal and marine natural resources. Two contrasting archetypal regional cases of coral reef governance are used as case studies. Both operate in similar institutional and sustainability contexts: a centrally coordinated marine protected area (MPA) in a northeastern Brazilian coastal region and a polycentric form of coastal and marine governance in an island archipelago in East Indonesia, both functioning in the context of governance decentralization. The article investigates how the social network characteristics associated with these contrasting forms of governance affect the respective governance networks' information transmission capacities and the robustness of their information transmission capacity under conditions of sociopolitical change. Stakeholder rosters (which are compiled from gray literature), meeting protocols, and interviews are used for a structured survey of MPA governance interactions among all identified parties. To compensate for missing data from about 20 percent of identified network actors, statements of other network actors on the network relations of the missing interviewees were included. The authors use the idea of reachability within the governance network to examine information transmission capacity. Network robustness is assessed by simulating the speed by which information transmission capacity decreases when the network actors with the highest closeness centrality are consecutively removed. The results show that the polycentric Indonesian governance network performed better than the centrally coordinated Brazilian MPA network in terms of both information transmission capacity and on robustness. The authors discuss the implications of this surprising result for the vulnerability of collaborative governance, combining theoretical deliberations with an analysis of their empirical data, in particular on differences in reachability. The presented approach of simulating the consecutive removal of central actors from the network and analyzing the impact of this on central network functions holds further promise for the analysis of environmental governance dynamics.

Theresa Schwenke and Eike Holzkämper present a bibliometric analysis of publications that address both environmental governance and social (-ecological) network analysis. Using a bibliometric network analysis approach, the authors identify a relatively small but rapidly growing set of publications that address both fields of study and identify the "intellectual linkages" between the identified subsets of publications. They describe in detail how they constructed both a citation network and a similarity network for the identified set of publications and explain, calculate, and interpret key metrics for both chosen network types. The presented analysis identifies the highest ranked articles within the citation network in terms of metrics for source, storage, and bridge functions. The analysis of the similarity network indicates the composition, frequency, clustering and similarity of different thematic focuses within the identified sets of publications on environmental governance and social (-ecological) network analysis. As a central underlying theme 
across all identified thematic focuses, the authors identify information, influence, and knowledge, all of which are manifestations of differences in power and knowledge production. Finding a prevalence of locally focused studies, they foresee more global level analysis for the future.

This article carefully explains how an innovative set of techniques-similarity network analysis and citation network analysis — was developed and applied and shows the complementarity of the chosen mix of methods. Pinpointing remaining shortfalls of their pilot study, such as its limited data base, the authors display how their approach increases future options for producing a more differentiated analysis of network types in environmental governance.

Ben Nagel presents a coastal case study from one of the most climate change vulnerable countries on earth, Bangladesh. In a rural community exposed to advancing soil salinity, he investigates the role of social networks in enabling households to use innovative production technologies to adapt to these new environmental conditions. The author examines how the character of relevant social networks, the position of a household within those networks, and a household's characteristics affect its adoption of innovative production techniques to adapt to more saline conditions. Contextualizing the case study via key informant interviews, focus discussion groups, and participatory wealth and poverty ranking exercises, the study uses a whole network household survey to characterize network actors and their connections in an explicitly locally grounded approach. With technology adoption as the dependent variable, the article maps information, labor, and money exchange networks and analyzes the network positions of actors of different demographic and wealth categories. This article includes a candid discussion of methodological biases and implementation hurdles relevant for those planning SNA interviews.

Adam Henry focuses on sustainability learning at the organizational level, addressing to what extent an organization's position in a larger environmental policy network determines learning outcomes. The author examines four hypotheses on the relations between bonding and bridging social capital, network segregation, and network expansiveness (degree centrality) with organizational learning outcomes in three regional land use and transportation policy networks in California. Policy network members are identified through archival research and a survey $(n=514)$, in which stakeholders (34 percent response rate) nominated collaborators and classified their own relations to them. Learning outcomes are assessed through stakeholder perceptions. With this data, three network types relevant to governance are constructed per study region: a trust, collaboration, and information exchange network. Regression modeling analyses, which treat organizations' network positions as independent and organizational learning as the dependent variable, are then implemented. Diverse and sometimes surprising results are discussed. While bonding social capital, belief segregation, and vertical segregation increased learning outcomes, functional domain segregation and brokerage (bridging capital 
measured by betweenness centrality) decreased learning. This pioneer paper on the effects of networks and network position opens the path to methodologically innovative, empirically based research on arguably one of the central preconditions for sustainable and effective environmental governance with the needed adaptive and transformative potentials: the learning outcomes for diverse decision-makers. Counterintuitive results such as those reported on the inhibitive effects of diversity and brokerage on learning call for more examples of carefully contextualized work to support network design that supports sustainability enhancing learning outcomes.

\section{Outlook}

We conclude with some thoughts arising from our reading of all the papers in this special issue.

\section{Network types and performance}

Networks are an analytical tool. In research, each network is a heuristic device, conceptualized and bounded for a particular purpose. The networks analyzed in the contributions to this special issue focus on diverse aspects of environmental governance including innovation adoption in agriculture, influence and power characteristics of social networks in water governance, social-ecological network research, and knowledge production. With the exception of Schwenke and Holzkämper (who focus on authors and publications in citation networks), all contributions analyze governance networks at a subnational regional or local level, with a medium number of involved actors. Work on multilevel local to global environmental governance networks is, with few exceptions (e.g., Gerhardinger et al., 2018), as yet not well developed.

Social network types, and how their features might be conditioned by the characteristics of surrounding system environments, needs to be further explored. Not only the social realm (i.e., differing approaches to governance) but also some of the geo-bio-physical features of the system to be governed are likely to affect the character and performance of a governance network. Gorris and Glaser (this issue) find, perhaps not accidentally, polycentric governance for an MPA that stretches across a physically rather loosely and incompletely connected island archipelago (in Indonesia), but centrally coordinated governance for an MPA that spans along a $150 \mathrm{~km}$ stretch of (Brazilian) coast connected by a good road. Better physical communication options may have led to more centralization in the Brazilian MPA and may affect information transmission capacities. Whether and under which circumstances such geophysical or social circumstances affect governance outcomes and/or the structure and organization of associated governance networks requires further explicitly interdisciplinary social-ecological research. 
Interviewees in social network studies are often unable or unwilling to discuss obstructive relations (e.g., Nagel, this issue). This may be a feature of their culture or due to the power or dependency relations interviewees see themselves as embedded in. Social network analyses are therefore likely to be better at identifying network functions and features that enhance desirable outcomes, but are likely to underrepresent social network features that reduce or obstruct them. Particularly in the environmental governance field, research designs will need to take this into account.

\section{Network states and dynamics}

The great majority of studies present the structure or state of a social network with point-in-time data (e.g., Nagel, this issue). Such "snapshot" representations of social networks that relate to specific time periods or points in time are useful for presenting network structures and comparing their characteristics via an increasing number of SNA metrics. This field of investigation has generated important new knowledge for environmental governance over the past two decades. Thus, for instance, Gorris et al. (2019) and Gorris and Glaser (this issue) present a comparative analysis on different governance network structures and discuss the resulting capacities of governance networks. What such work on network states has generally lacked to date is the temporal dimension. Some of the important questions for environmental governance that require knowledge of change over time are: Are there classical cycles in environmental governance network development and what drives them? How do networks change in response to crisis and how can this be influenced? What role does leadership change play in governance networks and how can this be affected?

Mancilla García and Bodin (this issue) and a number of other authors interested in research on social-ecological systems processes argue that there are only a few longitudinal studies that cover governance structures at different moments in time (Mancilla García et al., 2020).

More studies with an explicitly temporal dimension are needed. Longitudinal social network studies require much time and money, however, and to our knowledge, few if any have been published. Beyond the important work on network characteristics, the ability to analyze and envisage network change over time therefore needs to be enhanced to inform environmental governance and management. Authors in this special issue such as Corrêa et al. engage with this. Future work might include:

1. Connecting a series of point-in-time studies on the state and characteristics of networks to generate a temporal dimension.

2. Identifying network features that indicate the character and or the direction of network change processes. A concentration on network processes and their effects, rather than on the characteristics of networks at a point in time, has the potential to add the required temporal dimension. In line with Glaser et al. (2012), a set of generic indicators on network processes might be developed for this purpose. 
SNA has provided information on the character and structure of interactions between actors in environmental governance and management at particular points in time, or for short periods (e.g., Gorris, 2015). This enables the empirical investigation of theoretical concepts, such as multilevel governance or network robustness, and of related questions, for instance on the role of intermediaries, brokers, or institutional entrepreneurs in environmental governance and associated social learning. Classical SNA uses questionnaires that generate standardized network data for the calculation of quantitative metrics. SNA can either assess the relations of single actors, in ego networks, or of all actors to all others in a conceptualized closed universe. The approach finds its limits when larger periods of time (more than one or two years) are the focus of attention.

Another as yet only partly addressed question is how and under which circumstances agency is able to alter networks and thus affect both the influence of individual network actors and the actual environmental outcomes of governance and management efforts. Mancilla García and Bodin (this issue) provide important inputs here.

\section{Limits of network studies}

Network surveys are important tools for exploring and improving communication, coordination, and knowledge exchange. With larger networks, surveys meet their limits in terms of the attention span and time required by both interviewer and interviewee, and the number of interviews that need to be agreed to by network members and actually conducted tends to exceed the capacities of all but very large and well-staffed projects. Additionally, with rising network size, reporting errors and omissions increase. Recent fieldwork (Gorris et al., 2019; Nagel, this issue) indicates that beyond a certain network size, a total network survey will suffer unacceptable data quality problems as the attention span and capacities of both interviewer and participants are increasingly overstretched. In small networks, on the other hand, ethical problems arise with the difficulties of guaranteeing anonymity to interviewees. Networks with a medium number of actors (about 100) therefore hold the highest potential for generating reliable data in an ethically sound manner.

Active participation of non-academic stakeholders in the formulation of research questions, in the design of research methods, and in the discussion of results is important in environmental governance network analysis, as in other sustainability fields: given the importance of informed consent and trust between researchers and other participants, such participation will improve research implementation and use of results, perhaps more so in the collaboration-focused field of SNA than in other research fields. 


\section{New conceptual focuses in network analysis}

Homophily holds potential as an umbrella concept in the debate on governance for sustainable human-nature relations. It describes the tendency of those that share certain traits (such as education, race, or beliefs) to engage in relations with each other. In contrast to its opposite (heterophily), homophily has popularly been described as the propensity of "birds of a feather to flock together" and has been found to foster internal collaboration but also to inhibit wider consensus-building. Two articles in this special issue employ the homophily concept. Henry cites numerous sources that find belief homophily to be a strong force in generating segregated networks, which are reinforced by cognitive biases that support the (re)interpretation of any evidence to confirm prior convictions. Various studies (cited in Henry, this issue) find the resulting self-reinforcing belief-specific networks to inhibit learning. Contemporary belief-specific "communication bubbles" on the internet may be interpreted to demonstrate learning capacities for belief-specific networks. In the paper presented here, however, Henry presents results that show belief-oriented segregation in collaboration networks increases learning outcomes while greater belief diversity actually decreases it. The context in which this was found clearly matters, not least for policy design. We refer readers to the article for details on this interesting and surprising finding. Corrêa et al. (this issue) also employ the homophily concept. They distinguish self-organized and self-directed homophilic and heterophilic network visions of key local environmental managers and discuss the implications of these visions for diverse aspects of network performance. Here, the use of the homophily concept helps to identify the potential of local environmental managers as leaders and shapers of governance and management. Overall, the homophily concept in the environmental governance field emerges as a useful tool for exploring how diversity and homogeneity between network actors along various lines affect governance outcomes.

Various contributions stress the importance of linking the social and the ecological dimensions in environmental governance research and, of course, network approaches offer possibilities here. Scotti et al. (this issue) show that interactions which link variables of the human and the ecological domains have to be considered and that a balanced integration of both variable types is possible in loop analysis modeling.

Social-ecological networks is a concept that extends the purely social science focus of SNA into the natural science realm and vice versa. Schwenke and Holzkämper (this issue) show the emergence of network analysis in the environmental governance field. Kluger et al. (2020) differentiate three types of social-ecological network that are integrated in different ways and to different degrees and show how each is suited for a particular set of purposes. A novel approach, namely that of considering ecosystems and ecosystem components as actors in otherwise social networks 
(proposed by T. Schwenke, several personal communications, 2020), opens up new potential for using the network lens in the co-analysis of interrelated social and ecological dynamics.

\section{Methodological innovations}

\section{Mixed methods}

Mixed method approaches that use a network lens for the analysis of social-ecological systems and their governance have synergy potentials that merit attention. Recent methods and tools that complement classical SNA tools are agent-based modeling of social or social-ecological networks, participatory modeling, net-mapping, and social-ecological network modeling based on the building block approach. In its more radically system-based approach, loop analysis (Scotti et al., this issue) enables a fairly rapid identification of connections between drastically different variables in openly conceptualized complex system models. Structural equation modeling offers further potentials for a fine-grained analysis of causality in multidimensional complex dynamic systems. SNA, on the other hand, is firmly rooted within the social realm: it is therefore weaker on broader system-wide analysis, while strong in pinpointing the particular features and impacts of different network types on governance outcomes.

Mixing methods can also enable triangulation. SNA combined with net-mapping can highlight the differences between self-reported networks and stakeholder-specific perceptions of such networks. New options for the quantification of intrinsically qualitative net-mapping data can avoid the often immense time and effort required to implement full SNA surveys, especially in larger networks, and to develop novel approaches to whole network analysis. New avenues to unravel causality in network analysis, such as between network position, household traits, and engagement with new climate change adaptation techniques (Nagel, this issue) may benefit from further triangulation, such as on network data with data from social experiment work (e.g., Schlüter \& Vollan, 2015).

\section{New tools}

Diverse software has been used by the authors in this special issue. For analyzing SNA metrics, all authors (Nagel, Gorris \& Glaser, García \& Bodin, Corrêa et al.) used UCINET, which is probably the most complete tool for SNA measures and is relatively cheap (from about USD40). For visualizing the networks, Nagel, and also Gorris and Glaser, used Gephi, a free tool whose network design is often thought more appealing than NetDraw, which comes in the UCINET package. Due to their specific networks, Schwenke and Holzkämper used the free CitNet Explorer, 
which was developed for visualizing and analyzing citation networks of scientific publications, and the data mining software RapidMiner Studio. Henry used the statistical software $\mathrm{R}$ for data management and descriptive analysis, including network visualization, and Stata 12 for regression models. Scotti et al. conducted a pure qualitative analysis for modeling relations between factors. Although most authors stress the importance of the analysis of additional qualitative data for their work, be it qualitative interview data, focus group discussions, or similar, these are not given the same importance in describing the analysis, methods, and software tools used, and in the presentation of results. This may be due to the fact that SNA is originally a quantitative method, but the relative importance attributed to qualitative and quantitative data in network analysis could be more balanced in future research.

\section{Contribution of this special issue to environmental governance}

The contributions to this special issue are highly diverse in their methods and in the aspects of social-ecological systems they examine. Trends in the development of methods for environmental governance are not apparent. We do, however, present a number of ideas to inspire innovative method development for environmental governance research.

Adaptive management has proven a key approach for sustainability-focused environmental governance (Armitage et al., 2009; Berkes, 2009; Folke et al., 2005). The analytical approaches and associated methods presented here can help to further develop and adapt governance structures and processes in this endeavor: for instance, by examining the importance of specific actors (e.g., nongovernmental organizations) as leaders for change and innovation, or the importance of diverse ties for knowledge distribution and sharing. Insights on information flows that trigger social learning and influence can support social-ecological transformation: for instance, through fostering innovations or resilience in the face of adverse social, political, or environmental change. The case studies presented here focus on the local or regional governance level, but they also indicate how to expand analysis toward addressing multilevel governance. All this may lead to practical insights for policy-makers.

Also, while networks tend to concentrate on actors and their relations, it is time to move beyond "internally focused" analysis and go a step further, by looking at the effects of structures and agency on environmental governance, as well as at causalities. This could be done by, for example, combining methods, like SNA with choice or economic experiments. 
Network research tends to focus on either the social or the ecological side (Kluger et al., 2020); for instance, SNA on social actors. Some of the approaches presented here, mainly social-ecological network analysis and loop analysis, enable a better integration of ecological with social system dynamics. Network concepts in the governance field include the concept of a functionally oriented or sector-specificusually informal and closed-policy network, which contrasts with the more inclusive, democratic slant of the governance network concept (Blanco et al., 2011). Clearly both network types need to be distinguished and analyzed in the environmental governance field. Recent research further stresses the importance of informal network structures for policy-making (Suyo et al., 2020).

The methods presented here open opportunities for the integration of different views, thought styles, and knowledge, as they include participatory elements. These, however, may still be deepened and extended. Through their integration potential across boundaries of different kinds, social-ecological network analysis methods in particular may also serve to connect different scientific fields both with each other and with the science-policy interface, and thus to coproduce knowledge in a more integrative manner (Mollinga, 2010; Star, 2010).

This special issue provides knowledge on different methods in environmental governance. We hope that it inspires researchers to think outside their own disciplinary boxes, to cross methodological boundaries, and last, but not least, to engage in combining and further developing some of the diverse methodological options discussed here.

\section{Acknowledgments}

This special issue resulted from a symposium on "Generating knowledge on networks in environmental governance" at the SHE XXIII conference, Navigating Complexity: Human-Environmental Solutions for a Challenging Future, in Lisbon, Portugal, July 7-10, 2018.

We would like to thank all participants for contributing to the session and all authors in this special issue for submitting their work. We also thank all anonymous reviewers for their time spent on formulating their critical and constructive feedback.

We further acknowledge the funding that has made the editing of this special issue possible: BS was supported by the German Federal Ministry for Education and Research (Bundesministerium für Bildung und Forschung-BMBF) through a grant for the PlanSmart research group (grant no: 01UU1601B); MG's work was contributed via the project Network Analysis for Coastal and Marine Governance Project (SEN-MPA) (www.leibniz-zmt.de/en/research/research-projects/ sen-mpa.html). 
Finally, we thank Human Ecology Review, Robert Dyball, Ngaire Kinnear, and the whole team for their continuous support and kind collaboration.

\section{References}

Armitage, D. R., Plummer, R., Berkes, F., Arthur, R. I., Charles, A. T., Davidson-Hunt, I. J., ... Wollenberg, E. K. (2009). Adaptive co-management for social-ecological complexity. Frontiers in Ecology and the Environment, 7(2), 95-102. doi.org/10.1890/ 070089

Berkes, F. (2009). Evolution of co-management: Role of knowledge generation, bridging organizations and social learning. Journal of Environmental Management, 90(5), 16921702. doi.org/10.1016/j.jenvman.2008.12.001

Blanco, I., Lowndes, V., \& Pratchett, L. (2011). Policy networks and governance networks: Towards greater conceptual clarity. Political Studies Review, 9(3), 297-308. doi.org/ 10.1111/j.1478-9302.2011.00239.x

Folke, C., Hahn, T., Olsson, P., \& Norberg, J. (2005). Adaptive governance of socialecological systems. Annual Review of Environment and Resources, 30, 441-473. doi.org/ 10.1146/annurev.energy.30.050504.144511

Gerhardinger, L. C., Gorris, P., Gonçalves, L. R., Herbst, D. F., Vila-Nova, D. A., De Carvalho, F. G., ... Glavovic, B. C. (2018). Healing Brazil's Blue Amazon: The role of knowledge networks in nurturing cross-scale transformations at the frontlines of ocean sustainability. Frontiers in Marine Science, 4, Article 395. doi.org/10.3389/fmars.2017.00395

Glaeser, B., Bruckmeier, K., Glaser, M., \& Krause, G. (2009). Social-ecological systems analysis in coastal and marine areas: A path toward integration of interdisciplinary knowledge. In P. Lopes \& A. Begossi, Current Trends in Human Ecology (pp. 183-203). Cambridge Scholars Publishing.

Glaser, M., Christie, P., Diele, K., Dsikowitzky, L., Ferse, S., Nordhaus, I., ... Wild, C. (2012). Measuring and understanding sustainability-enhancing processes in tropical coastal and marine social-ecological systems. Current Opinion in Environmental Sustainability, 4(3), 300-308. doi.org/10.1016/j.cosust.2012.05.004

Gorris, P. (2015). Entangled? Linking governance systems for regional-scale coral reef management: Analysis of case studies in Brazil and Indonesia. [Doctoral dissertation, Jacobs University and Leibniz Centre for Tropical Marine Research (ZMT)]. IRC-Library, Information Resource Center der Jacobs University Bremen. nbn-resolving.org/urn:nbn:de:gbv:579opus-1002112

Gorris, P., Glaser, M., Idrus, R., \& Yusuf, A. (2019). The role of social structure for governing natural resources in decentralized political systems: Insights from governing a fishery in Indonesia. Public Administration, 97(3), 654-670. doi.org/10.1111/padm.12586 
Grimble, R., \& Wellard, K. (1997). Stakeholder methodologies in natural resource management: A review of principles, contexts, experiences and opportunities. Agricultural Systems, 55(2), 173-193. doi.org/10.1016/S0308-521X(97)00006-1

Kluger, L. C., Gorris, P., Kochalski, S., Mueller, M. S., \& Romagnoni, G. (2020). Studying human-nature relationships through a network lens: A systematic review. People and Nature, 2(4), 1100-1116. doi.org/10.1002/pan3.10136

Mancilla García, M., Hertz, T., Schlüter, M., Preiser, R., \& Woermann, M. (2020). Adopting process-relational perspectives to tackle the challenges of social-ecological systems research. Ecology and Society, 25(1), Article 29. doi.org/10.5751/ES-11425-250129

Mollinga, P. (2010). Boundary work and the complexity of natural resources management. Crop Science, 50(S1), S-1-S-9. doi.org/10.2135/cropsci2009.10.0570

Phillipson, J., Lowe, P., Proctor, A., \& Ruto, E. (2012). Stakeholder engagement and knowledge exchange in environmental research. Journal of Environmental Management, 95(1), 56-65. doi.org/10.1016/j.jenvman.2011.10.005

Reed, M. S. (2008). Stakeholder participation for environmental management: A literature review. Biological Conservation, 141(10), 2417-2431. doi.org/10.1016/j.biocon.2008. 07.014

Schlüter, A., \& Vollan, B. (2015). Flowers and an honour box: Evidence on framing effects. Journal of Behavioral and Experimental Economics, 57, 186-199. doi.org/10.1016/j.socec. 2014.10.002

Star, S. L. (2010). This is not a boundary object: Reflections on the origin of a concept. Science, Technology, \& Human Values, 35(5), 601-617. doi.org/10.1177/0162243910377624

Suyo, J. G. B., Le Masson, V., Shaxson, L., Luhan, M. R. J., \& Hurtado, A. Q. (2020). A social network analysis of the Philippine seaweed farming industry: Unravelling the web. Marine Policy, 118, Article 104007. doi.org/10.1016/j.marpol.2020.104007 
This text is taken from Human Ecology Review, Volume 26, Number 2, 2020, published by ANU Press, The Australian National University, Canberra, Australia. doi.org/10.22459/HER.26.02.2020.01 